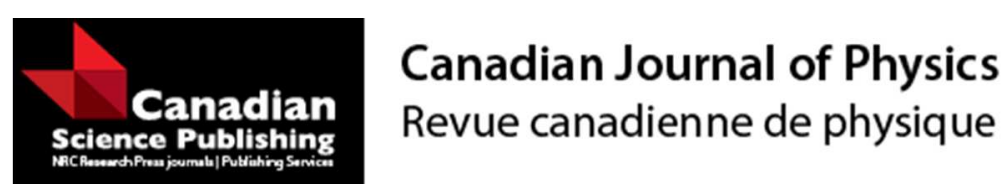

\title{
Time-dependent potential functions to stretch the time distributions of ion pulses ejected from EBIST
}

\begin{tabular}{|r|l|}
\hline Journal: & Canadian Journal of Physics \\
\hline Manuscript ID & cjp-2016-0716.R1 \\
\hline Manuscript Type: & Article \\
\hline Date Submitted by the Author: & $18-$ Dec-2016 \\
\hline Complete List of Authors: & Lapierre, Alain; National Superconducting Cyclotron Laboratory, \\
\hline Keyword: & $\begin{array}{l}\text { Electron beam ion source, highly charged ions, charge breeder, ion traps, } \\
\text { radioactive isotopes }\end{array}$ \\
\hline \multicolumn{2}{|l}{} \\
\hline
\end{tabular}




\title{
Time-dependent potential functions to stretch the time distributions of ion pulses ejected from EBIST
}

\author{
A. Lapierre \\ National Superconducting Cyclotron Laboratory, \\ Michigan State University, \\ 640 South Shaw Lane, \\ East Lansing, 48824, USA
}

Phone: $517-908-7675$

Fax: $517-353-5967$

E-mail: lapierre@nscl.msu.edu

PACS: 07.77.Ka, 37.10.Ty

\begin{abstract}
Electron beam ion sources and traps (EBIST) produce and trap highly charged atomic ions with an electron beam of high current density. The ions are confined in the radial space-charge potential of the electron beam and a long square-shaped axial electrostatic potential well. An important field of application of EBIST is charge breeding of highly charged ions at radioactive ion beam facilities. There, highly charged radioactive isotopes are accelerated by particle accelerators for experiments in nuclear astrophysics and to study the structure of unstable nuclei. The width in time of the ion pulses ejected from EBIST can often contain too many ions for nuclear-physics detection systems to efficiently detect all single radioactive isotopes or related events. Neglecting the influence of ion-ion collisions on the extraction rate, this publication derives for different initial thermal energy distributions of the trapped ions the time-dependent trap-opening functions to stretch the time distribution of ion pulses ejected from an EBIST trapping potential for the release of ions at a constant rate over an extended extraction period.
\end{abstract}




\section{INTRODUCTION: EBIST AND THEIR EJECTED ION PULSES}

Electron beam ion sources and traps (EBIST) produce and confine highly charged atomic ions with the use of an electron beam of high current density ${ }^{1-3}$. In these devices, as depicted in Fig. 1, a high-current electron beam is first generated by an electron gun. The electron beam is then compressed to high density as it is injected into a strong magnetic field provided by a solenoid or Helmholtz coils (usually) and enters a series of coaxial cylindrical electrodes located in the magnet bore. There, the highly charged ions are produced by the electron beam, which ionizes injected atomic gas or ions by successive electron-ion collisions. The highly charged ions are confined by the negative space-charge potential of the electron beam in the radial direction and two positive electrostatic potential barriers forming an approximate square-shaped potential well in the axial direction. After passing through the trap's center, the electron beam is stopped in the electron collector.

EBIST can provide beams of highly charged ions with unique properties (e.g., small energy spread and transverse emittance, and with few contaminants). As a consequence, one important application, which has grown over the last decade, is as charge breeder of rare isotopes at radioactive ion beam facilities ${ }^{4-9}$. At such facilities, EBIST are utilized to convert singly charged radioactive ions into highly charged ions for subsequent acceleration by a particle accelerator. The ions are injected and ejected as beams by passing through the collector. As shown in Fig. 2, singly charged ions are first typically injected as ion pulses, charge bred to a desired charge state, and then extracted. After acceleration by the accelerator to high beam energies, which can only be reached with the aid of high charge states, the radioactive isotopes are transported to experimental stations for experiments in nuclear physics. Stable isotopes are normally abundant and can, thus, be ejected out of EBIST as continuous beams with affordable losses in breeding efficiency inherent to this ejection method. However, rare isotopes are only produced in minute quantities and, in addition, can have very short half-lives. The most efficient charge breeding method is then to periodically inject and extract pulsed beams where a single highly charged ion pulse is ejected after each charge breeding time period. A simple scheme to eject ion pulses from EBIST, and fully empty the content of the trap, consists of opening it by lowering the voltage applied to the trap's potential barrier located on the collector side (named here the extraction potential barrier) with a step function in time. The width of the time distribution 
of the ions ejected using this scheme and reaching a particle detector is typically in the range of tens of microsecond $(\mu \mathrm{s})$. In several cases, however, the number of ions within the width of these pulses (i.e., the instantaneous rate) can overcome the detection system being used and prevent efficient detection of all single radioactive ions or related events within each pulse. To go around this limitation, the time distribution of the ejected ions has to be adequately spread in time to control their arrival time to the detector. This is to ensure that each ion does not hit the detector within a 'dead' time, during which the detection system is busy processing previously detected ions and, hence, not able to record another event. The release of ions can be distributed over an extended time period by opening the ion trap over tens of millisecond (ms) with a continuous function resembling a ramp. In an ideal time distribution, the ejected ions have to be dispersed as evenly as possible in time. One can ask: how should the extraction barrier potential of a long square-shaped electrostatic potential well be lowered as a function of time for releasing ions at a constant rate over an extended extraction period and generating a flat-top (square) ion distribution? And what are the time-dependent functions to do so for different initial thermal energy (probability density) distributions of the trapped highly charged ions? This publication attempts to answer these questions by neglecting the effect of ion-ion collisions on the rate of ion extraction. Neglecting ion-ion collisions in the ejection rate implies that this rate depends only on the opening rate of the trap, which is a valid assumption as long as the extraction time is significantly shorter than the collision time as for low ion densities. To illustrate with an example, the number of rare-isotope ions (e. g., $\left.{ }^{34} \mathrm{Ar},{ }^{37} \mathrm{~K},{ }^{47} \mathrm{~K}\right)$ injected into the ReA EBIST ${ }^{7}$ is typically less than $5 \times 10^{5}$ ions/s. Breeding charge states in the vicinity of $q_{i}=10$ requires injection and extraction of ion pulses at a rate of approximately $10 \mathrm{~Hz}$, corresponding to $5 \times 10^{4}$ ions per injected pulses. Assuming 4 times more ion contaminants from residual gas, one can estimate a trapped ion number density of $\sim 3 \times 10^{6}$ ions $/ \mathrm{cm}^{3}$ per charge breeding cycle. The ion-ion Coulomb collision rate is directly proportional to the ion density ${ }^{1}$. For that low ion density, before the start of the extraction process, the ion-ion collision time constant is approximately a second. Based on numerical simulations, this collision time is expected to remain within the same scale over an ejection time of less than 100 ms. During ejection, the time constant of ion escape out of the trap induced by ion-ion collisions ${ }^{1}$ is estimated to remain longer than hundreds of ms for a wide range of varying extraction barrier potentials. These time constants are longer than the ejection 
time constant of the time-dependent functions investigated in the present work. The simple collision-free model presented here is expected to be mostly valid for ion number densities of less than $\sim 10^{6}$ ions $/ \mathrm{cm}^{3}$ with charge states in the vicinity of $q_{i}=10$ for extraction times of less than about $100 \mathrm{~ms}$. Obviously, beyond these ranges, this simple model can still be used to calculate or generate time-dependent functions for ion ejection, but the extracted ion time distributions may show prominent features resulting from ion-ion collisions influencing the rate of extraction. For instance, this rate may be higher at the beginning of the ejection time when the trapped ion density is high and may become more uniform toward the end as it decreases.

To the author's knowledge, there exists no theoretical work on the time structure of ion pulses extracted from EBIST. Charge breeding simulations with a static trapping potential, including an ion escape rate derived from the Fokker-Planck equation, have been performed by several EBIST groups ${ }^{10-14}$. Despite that, no time-dependent calculations have been devoted to investigating the time structure of the ejected ion pulses following a dynamic opening of the trap. Time-dependent calculations involving ion extraction from an EBIT were conducted by Marrs ${ }^{12}$ for studying the effect of self-cooling on the beam emittance by dynamically opening the trap over a long time scale. However, Marrs did not study the time distribution of the extracted ions. Note that in this work, ions released independently of ion-ion collisions, when the extraction barrier potential is lowered below their kinetic energies in the trap, appear to have not been taken into account. As the trap is dynamically opened, this collision-free process dominates for low trapped ion densities.

\section{TIME DISTRIBUTIONS OF IONS EJECTED WITH A STEP FUNCTION}

Ions confined in the vicinity of two adjacent electrodes biased at different voltages are subject in the longitudinal $(z)$ direction to a quadratic potential ${ }^{15}$. However, ions trapped in a long square-shaped electrostatic potential well, as illustrated in Fig. 2, are affected by such a potential near the potential barriers, and experience, to a good approximation, a constant potential over the entire length of the trapping region. The width of the time distribution of ion pulses ejected from such a long trap obtained by lowering the extraction barrier with a step time function can be considered to be dominated by three main elements: the distribution in polar angle $(\phi)$ of the velocity $\mathbf{v}_{\mathbf{i}}$ of the trapped ions with respect to the 

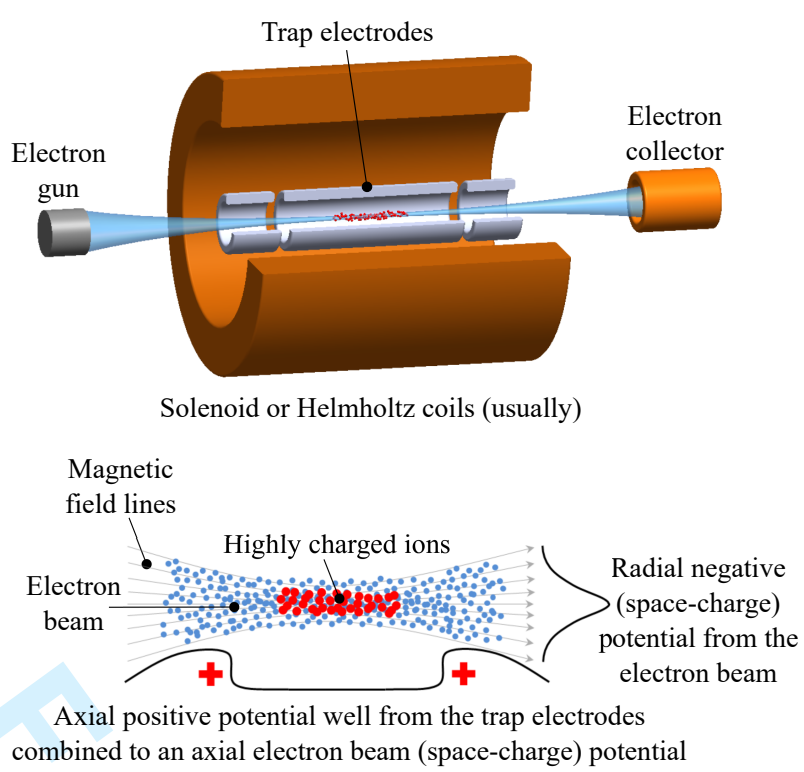

FIG. 1. Simplified model and cartoon of an electron beam ion source, which consists of three basic components: 1) an electron gun providing a high-current electron beam, 2) an ion trap made with coaxial electrodes sitting in the bore of a solenoid or coils (usually), used to generate a magnetic field of high flux density, and 3) a collector to stop the electron beam. The electron beam is magnetically compressed in the trap's center to a high current density, needed for production of highly charged ions by electron-impact ionization.

axis of ion ejection $z$, which is along the magnetic field axis; their speed distribution; and the length of the trapping potential. Upon opening the trap, the number of ejected ions, $N_{i}^{e j}$, of a given ion species $i$ can be expressed as

$$
N_{i}^{e j}=\frac{N_{i}^{t o t}}{2 \pi L} \int_{0}^{2 \pi} \int_{0}^{v_{i}^{\max }} \int_{0}^{L} f\left(v_{i}\right) d l d v_{i} d \phi
$$

where $N_{i}^{t o t}$ is the total number of ions and $f\left(v_{i}\right)$ is their speed distribution normalized to 1 . $v_{i}^{\max }$ tends toward infinity. $L$ is the length of the trapping region. The distribution in polar angle of the ion velocity is assumed isotropic, and by rotational symmetry, the distribution in azimuthal angle $(\theta)$ is assumed uniform around the $z$-axis. The polar angle is the angle between the magnitude of the ion velocity, $\left\|\mathbf{v}_{\mathbf{i}}\right\|=v_{i}$, and its component along the $z$-axis, $v_{i z}$, such that $\phi=\arccos \left(v_{i z} / v_{i}\right)$, where $v_{i z}=l / t$ in which $l$ is the distance traveled by the ions being released before exiting the trapping region and $t$ is their time of flight to the trap's exit. The exit is the inner edge of the extraction barrier defined as the origin of the 
a) Injection, capture, breeding, and ejection sequence

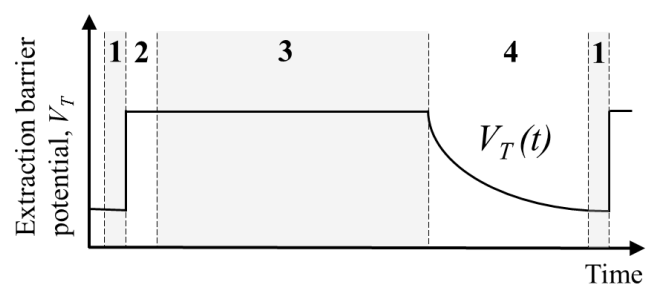

Time distribution of the (spread) ejected ion pulses

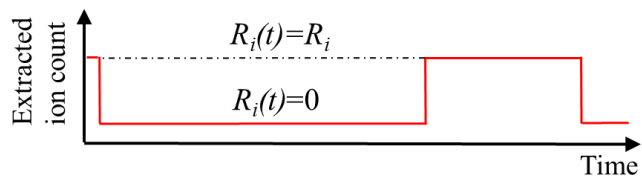

1. Injection of a singly charged ion pulse

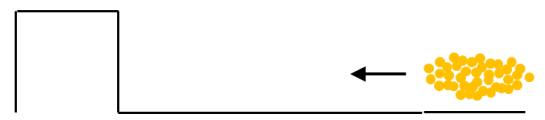

2. Capture Trapping potential barriers

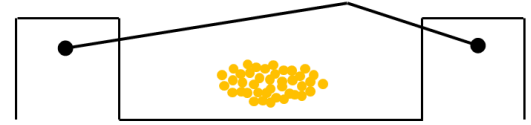

3. Charge breeding of highly charged ions

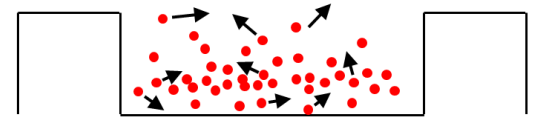

4. Slow ion ejection

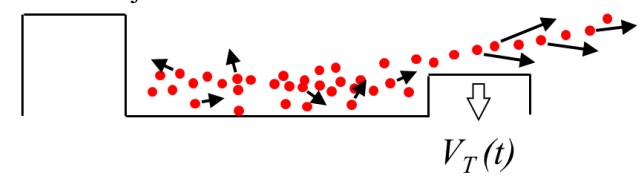

b)

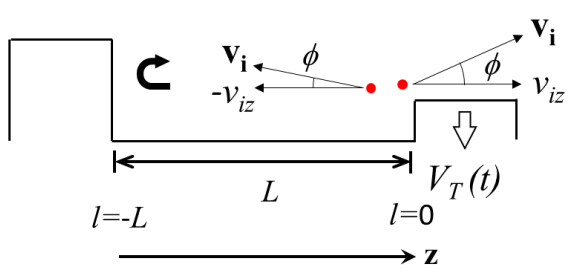

FIG. 2. a) Pulsed ion beam injection and extraction scheme: 1. singly charged ions are injected, 2. captured in the axial trapping potential, 3. charge bred to highly charged ions with a MaxwellBoltzmann energy distribution (the arrows show the ions' velocity vectors), and 4 . released at a constant rate, $R_{i}$, by slowly lowering the collector-side trapping barrier potential with a continuous (time-dependent) function. b) Velocity vector components of two trapped ions in a trap of length $L$. 
$z$-axis where $l=0$ (see Fig. 2). It is important to stress that for a uniform magnetic field over the entire length of the trap (including the trap's exit at the origin $l=0$ ), as it is assumed here, the magnetic field does not influence the flight time of the ions as it depends only on the ion velocity component $v_{i z}$, parallel to the field axis $\left(\mathbf{B}=B_{z} \hat{\mathbf{z}}\right)$. The Lorentz force the magnetic field exerts on the ions $\left(\mathbf{v}_{\mathbf{i}} \times \mathbf{B}\right)$ is thus null along the magnetic field axis. In practice, however, the ejected ions having initial thermal energy stored in their radial motion have the radial energy converted into axial energy in experiencing the magnetic field gradient when extracted toward a detector in a low-field region ${ }^{16}$. The acceleration energy of EBIST beams is normally greater than tens of keV. The addition of the thermal energy of the ions being ejected to the acceleration energy slightly reduces the ions' flight times to the detector. Nevertheless, this has a negligible effect on their time distribution on the ms time scale. Therefore, replacing $d v_{i z}$ with $-l / t^{2} d t$ for a fixed ion position $l, d \phi$ can be expressed as

$$
d \phi=\frac{1}{\sqrt{1-\left(\frac{l}{v_{i} t}\right)^{2}}} \frac{l}{v_{i} t^{2}} d t .
$$

The ions having a negative $z$-component of their velocity vector with respect to the ejection direction have on average longer flight times to the trap's exit then the ones having a positive component. For instance, an ion at a position $l=0$ having a negative velocity vector $z$ component must travel twice the trap length $(L)$ to exit the trap; it must first travel to the electron-gun side to be reflected off the potential barrier before traveling back to the collector-side barrier (see b) in Fig. 2). It is interesting to re-write Eq. (1) as the sum of two components having positive and negative velocity $z$-vectors. The positive component can be obtained by substituting $d \phi$ (Eq. (2)) into Eq. (1). The negative component is deduced by replacing $l$ with $(-2 L-l)$ in Eq. (2), and substituting the new resulting $d \phi$ into Eq. (1):

$$
\begin{gathered}
N_{i}^{e j \oplus}=\frac{-N_{i}^{t o t}}{\pi L} \int_{0}^{\infty} \int_{0}^{v_{i}^{\max }} \int_{-L}^{0} \frac{f\left(v_{i}\right)}{\sqrt{1-\left(\frac{l}{v_{i} t}\right)^{2}}} \frac{l}{v_{i} t^{2}} d l d v_{i} d t \\
N_{i}^{e j \ominus}=\frac{-N_{i}^{t o t}}{\pi L} \int_{0}^{\infty} \int_{0}^{v_{i}^{\max }} \int_{-L}^{0} \frac{f\left(v_{i}\right)}{\sqrt{1-\left(\frac{-2 L-l}{v_{i} t}\right)^{2}}} \frac{-2 L-l}{v_{i} t^{2}} d l d v_{i} d t
\end{gathered}
$$

The time distribution of the ejected ions after the opening of the trap can be obtained by a differentiation of Eqs (3) and (4) with respect to time. Summing these two equations and 
integrating over the trap length one obtains the number of extracted ions within a time interval $d t$ :

$$
\frac{d N_{i}^{e j}}{d t}=\frac{N_{i}^{t o t}}{\pi L} \int_{0}^{v_{i}^{\max }} v_{i} f\left(v_{i}\right)\left[1-\sqrt{1-\left(\frac{2 L}{v_{i} t}\right)^{2}}\right] d v_{i} .
$$

The distribution in speed of the trapped ions can be a rather complicated function (e.g., Maxwell-Boltzmann distribution), and it is normally more convenient to solve Eq. (5) numerically or through the use of charged-particle trajectory simulations. However, to illustrate how the width of the time distribution of ejected ion pulses depends on the ion velocity and trap length, Eq. (5) can be simplified assuming that $f\left(v_{i}\right)$ is a $\delta$ function. Re-writing Eq. (5) making this assumption, one obtains:

$$
\frac{d N_{i}^{e j}}{d t}=\frac{N_{i}^{t o t} v_{i}}{\pi L} \Re\left[1-\sqrt{1-\left(\frac{2 L}{v_{i} t}\right)^{2}}\right] .
$$

Figure 3 shows time distributions calculated with Eq. (6) for two different trap lengths $(L)$ and three ion kinetic energies, $E_{i}\left(v_{i}\right.$ is obtained from the non-relativistic relation $v_{i}=$ $\left.\sqrt{(} 2 E_{i} / m_{i}\right)$, where $m_{i}$ is the ion mass). The calculations were performed for argon of atomic mass unit $A=40 \mathrm{u}$. The plateaus observed in the distributions correspond to the time period when $2 L>v_{i} t$ for which the square-root term in Eq. (6) is an imaginary number and the only real number within the square brackets is 1. For comparison, Fig. 3 also shows the result of a charged-particle trajectory simulation assuming a Maxwell-Boltzmann speed distribution (for three degrees of freedom), which can be seen, as expected, as the superposition of several time distributions obtained from Eq. (6) assuming different initial ion energies. The simulation was conducted with a magnetic field in the trap of $B=4 \mathrm{~T}$ for ions of argon of charge $q_{i}=1$ and an initial ion temperature in units of energy of $k_{B} T_{i}^{\circ}=50$ $\mathrm{eV}\left(k_{B}\right.$ is the Boltzmann constant and $T_{i}^{\circ}$ is the initial ion temperature). A charge of $q_{i}=1$ was used to simplify and accelerate the simulation, since less computing power is necessary to calculate the trajectories of the ions in the magnetic field. Note that the simulation was performed for $\phi_{\max }<85^{\circ}$ to prevent ions from being confined in the trap for infinitely long times (e.g., for $\phi_{\max }=90^{\circ}$ ). The primary conclusion to draw from these plots is for trapping regions of the order of a meter or more in length and ion kinetic energies of tens of eV, the trap length only affects the width in time of the ejected ion pulses by less than a ms. This indicates that for stretching the width of ion pulses ejected from a long trapping 


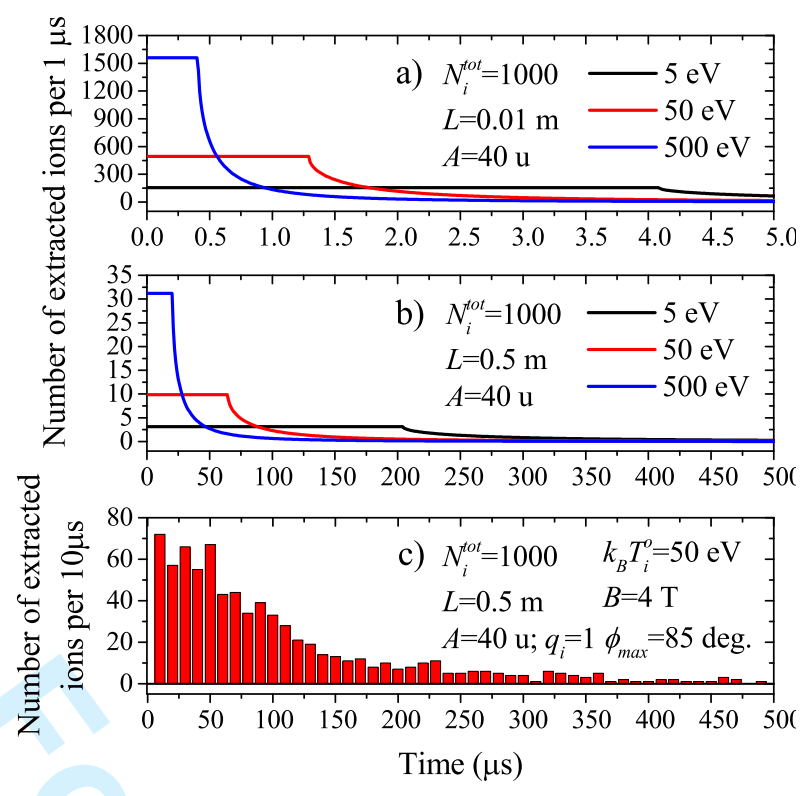

FIG. 3. Time distributions (calculated with Eq. (6)) of argon ion pulses ejected by lowering the extraction barrier potential with a step time function for a trap length of a) $L=0.01 \mathrm{~m}$ and b) $L=0.5 \mathrm{~m}$, and ion kinetic energies of 5,50 , and $500 \mathrm{eV}$. Note the difference in time scale between a) and b). For comparison, c) shows the result of a charged-particle trajectory simulation assuming a Maxwell-Boltzmann speed distribution (see text).

potential beyond a time range of a ms, the influence of the length of the ion trap on the time distribution of the ejected ions is negligible.

\section{TIME-DEPENDENT FUNCTIONS TO STRETCH THE TIME DISTRIBU- TIONS OF EJECTED IONS}

A simple extraction method for releasing ions consists of opening the trap by lowering the extraction barrier potential with a discontinuous time function (step function). However, if the detection system the ions are accelerated to can only detect one particle every $50 \mu \mathrm{s}$, for instance, while extraction with a step function releases thousands of ions within a $10-\mu$ s long pulse, the width of the ejected ion pulses must be spread in time to reduce the instantaneous rate. Here, the instantaneous rate is defined as the ratio of the number of extracted ions to the pulse width. This can be accomplished by lowering the extraction barrier potential with a continuous function over a long period of time. As seen in the previous section, for a pulse width being stretched by more than a ms, the length of the trapping region has a 
negligible contribution to the time distribution of the ejected ions. This distribution can then be considered to be defined only by the initial energy distribution of the trapped ions and the time function employed to lower the extraction barrier potential.

The total number of ions, $N_{i}^{t o t}$, is conserved during ion ejection and is the sum of the number of trapped, $N_{i}^{t r}$, and ejected, $N_{i}^{e j}$, ions:

$$
N_{i}^{t o t}=N_{i}^{t r}+N_{i}^{e j}
$$

The (instantaneous) variation in time of the number of ejected ions (i.e., the ion extraction rate) is obtained by differentiation of Eq. (7) with respect to time. $N_{i}^{\text {tot }}$ being a conserved quantity $\left(d N_{i}^{t o t} / d t=0\right)$, doing so yields:

$$
\frac{d N_{i}^{e j}}{d t}=-\frac{d N_{i}^{t r}}{d t}
$$

For an initial ion energy distribution $F\left(E_{i}\right)$, the number of trapped ions of energy $E_{i}$ within an infinitesimal energy interval $d E_{i}$ can be expressed as

$$
d N_{i}^{t r}=F\left(E_{i}\right) d E_{i}
$$

Note that, unlike in Section II in which $f\left(v_{i}\right)$ is normalized to $1, F\left(E_{i}\right)$ is normalized to the total number of ions $\left(N_{i}^{t o t}\right)$ to simplify the notation in the following equations. From this expression, the number of trapped ions can be obtained by integrating the energy distribution over $E_{i}$ :

$$
N_{i}^{t r}=\int_{0}^{E_{i}^{\max }(t)} F\left(E_{i}\right) d E_{i},
$$

where the upper integration limit $E_{i}^{\max }(t)$ is the maximum energy the trapped ions can gain without escaping the trapping potential. For the present studies, this maximum energy is time dependent. The variation in time of the number of trapped ions can then be expressed as

$$
\frac{d N_{i}^{t r}}{d t}=\frac{d}{d t}\left[\int_{0}^{E_{i}^{\max }(t)} F\left(E_{i}\right) d E_{i}\right] .
$$

Combining Eqs (8) and (11), and replacing $d N_{i}^{e j} / d t$ with $R_{i}(t)$, one can obtain a general expression describing the extraction rate as a function of the energy distribution of the trapped ions:

$$
R_{i}(t)=-\frac{d}{d t}\left[\int_{0}^{E_{i}^{\max }(t)} F\left(E_{i}\right) d E_{i}\right]
$$


The time distribution of the ions ejected by lowering the extraction barrier potential with a time-dependent function can directly be derived from Eq. (12). As an example, the extraction barrier potential can be decreased with a linear function such as $V_{T}(t)=-\alpha t+V_{T}^{\circ}$, where $\alpha$ is the rate at which the potential is changed and $V_{T}^{\circ}$ is the initial potential at $t=0$. In EBIST devices, the radial trapping potential provided by the electron beam space-charge potential is generally deeper than the axial potential created with the trap electrodes. The depth of the trap's potential well is then defined by the lower of the two axial potential barriers. During the charge breeding process, the trapped ions distribute themselves in the potential well through ion-ion collisions according to their kinetic energies, and can reach thermal equilibrium. They experience a trapping potential proportional to their charge, which, in the typical case that the extraction barrier of potential $\left(V_{T}\right)$ is the lowest, is equal to $q_{i} V_{T}(t)$. The ions in the trap having little kinetic energy are confined to the bottom of the potential well, while those of kinetic energy higher than the trapping potential can overcome it, escape, and, as a result, be ejected. The minimum kinetic energy of the ions being ejected, $E_{i}^{\max }(t)$, can then be expressed in dependence of the trapping potential as $E_{i}^{\max }(t)=q_{i} V_{T}(t)$. Replacing $E_{i}^{\max }(t)$ with $q_{i}\left(-\alpha t+V_{T}^{\circ}\right)$ in Eq. (12), one can obtain the ion extraction rate as a function of $V_{T}(t)$ :

$$
R_{i}(t)=\alpha q_{i} F\left(q_{i} V_{T}(t)\right)
$$

Equation (13) shows that the time distribution of ions ejected out a long square trapping potential with a linear function is proportional to the ion energy distribution and can even be used to measure it (for examples, see Refs ${ }^{17,18}$ ). Figure 4 presents time distributions obtained by substituting different ion energy distributions into Eq. (13). As explained in more detail in the next sections, in this figure, 1-DoF and 3-DoF stand for one and three degrees of freedom, respectively. $\sigma_{i}$ is the width of the Gaussian distribution and $E_{a v}$ is its average energy. Note that releasing ions by lowering the extraction barrier potential over a long time scale can adiabatically reduce the temperature of the trapped ions. As discussed earlier, for low ion densities, the ion-ion collision time can be significantly longer than the ejection time constant studied here of less than $100 \mathrm{~ms}$. In this special case, the evolving energy distribution of the trapped ions during ejection can be seen as the initial energy distribution being continuously truncated, only, by the release of ions as the ions in the trap do not have sufficient time to re-distribute their energy by ion-ion collisions and reach a 


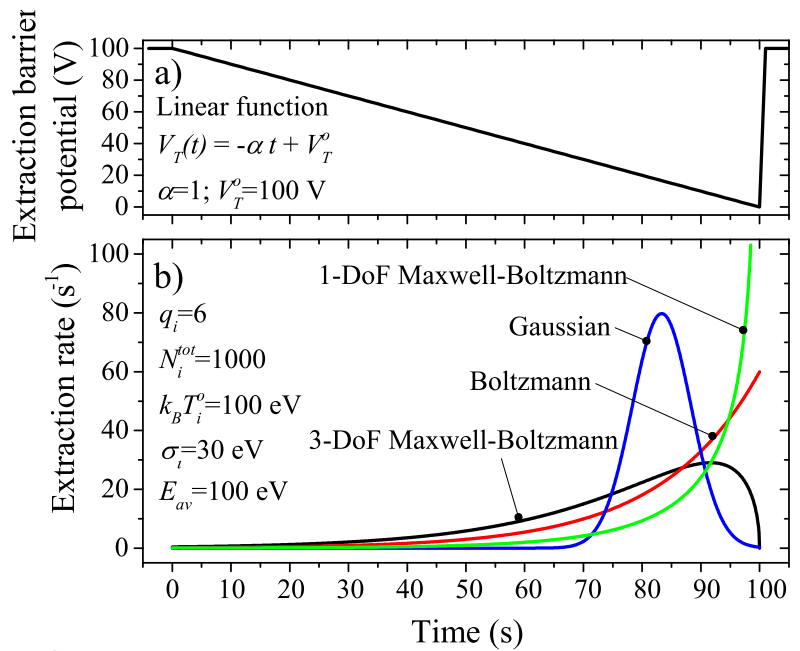

FIG. 4. Time distributions of ions ejected with a linear function for different initial ion energy distributions.

new thermal equilibrium. Hence, in Eq. (12) and in the following sections, it is assumed that during ejection the energy distribution of the remaining ions in the trap does not vary and remain unchanged with respect to the initial thermal energy distribution. Moreover, a related aspect is the gain in energy of the trapped ions by interacting with the electron beam (i.e., electron-beam heating ${ }^{1}$ ). For an electron-beam energy and current of $15.5 \mathrm{keV}$ and 400 $\mathrm{mA}$, respectively, and argon ions of a $q_{i}=10$ charge state, the electron-beam heating power is estimated to be approximately $120 \mathrm{eV}$ per ion per second. Over an ejection time constant of less $100 \mathrm{~ms}$, this corresponds to an average energy gain of the trapped ion ensemble of less than $12 \mathrm{eV}$. This can still be neglected in comparison with the initial temperature of the trapped ions, which, depending on operating parameters, is typically in the range of a few hundreds of eV.

In an ideal case, the release of ions can be uniformly distributed in time to extract a constant rate, $R_{i}$, over the entire extraction period to produce a square-like (or flat-top) ion distribution (as shown in Fig. 2). Hence, replacing $R_{i}(t)$ with $R_{i}$ in Eq. (12), and integrating, one obtains:

$$
R_{i} t=-\int_{0}^{E_{i}^{\max }(t)} F\left(E_{i}\right) d E_{i}+C^{\circ}
$$

where $C^{\circ}$ is a constant that can be obtained from the initial condition at $t=0 . \quad R_{i}$ is function of the lowering rate of the extraction barrier potential, which, to keep the ion 


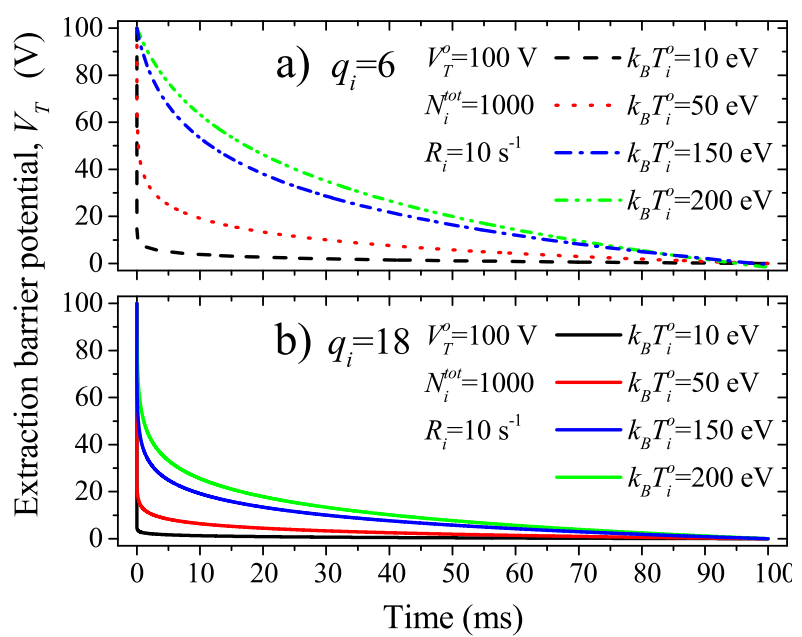

FIG. 5. Optimum functions to eject trapped ions having a Boltzmann energy distribution for different ion species a) $q_{i}=6$ and b) $q_{i}=18$, and initial ion temperatures.

extraction rate constant, has to vary with time to match the energy distribution of the trapped ions. In other words, for each time interval $d t$ the barrier potential is lowered, the energy distribution is truncated by an energy interval $d E_{i}$, releasing all ions within this energy range. The lowering rate must vary with time to modify the energy interval in order to keep the ejected number of ions constant. $R_{i}$ is the constant rate of ions transported to a detection system. It is an adjustable parameter, adjusted to keep the instantaneous rate lower than the maximum rate the detection system can handle by choosing an extraction period for a given total number of ions ejected per pulse. In the next sections, the optimum or ideal time-dependent functions for lowering the extraction barrier potential to provide a constant extraction rate are derived for four different assumed ion energy distributions.

\section{A. Boltzmann distribution}

For simplicity, one may assume that the energy distribution of the ions in the trap is well represented by a Boltzmann distribution normalized to the total number of trapped ions such as

$$
F\left(E_{i}\right)=\frac{N_{i}^{t o t}}{k_{B} T_{i}^{\circ}} \exp \left[-\frac{E_{i}}{k_{B} T_{i}^{\circ}}\right]
$$

where, as already mentioned above, $k_{B}$ is the Boltzmann constant and $T_{i}^{\circ}$ is the initial temperature of the ion ensemble. The optimum function to uniformly distribute the release 
of ions over an extended extraction period can be deduced by substituting Eq. (15) into Eq. (14), which can be re-written as

$$
\frac{R_{i}}{N_{i}^{t o t}} t=\exp \left[-\frac{E_{i}^{\max }(t)}{k_{B} T_{i}^{\circ}}\right]-1+C_{B}^{\circ},
$$

The maximum energy of the ions confined within the trapping potential is defined as $E_{i}^{\max }(t)=q_{i} V_{T}(t)$. Replacing $E_{i}^{\max }(t)$ in Eq. (16) and solving $V_{T}(t)$ analytically for the initial condition $V_{T}(t=0)=V_{T}^{\circ}$, one obtains:

$$
V_{T}(t)=-\frac{k_{B} T_{i}^{\circ}}{q_{i}} \ln \left[\frac{R_{i}}{N_{i}^{t o t}} t+\exp \left[-\frac{q_{i} V_{T}^{\circ}}{k_{B} T_{i}^{\circ}}\right]\right] .
$$

Figure 5 shows Eq. (17) plotted for different ion species and temperatures. In Fig. 5, $R_{i}$ was calculated from the ratio of the total number of ions $\left(N_{i}^{t o t}=1000\right)$ extracted in each pulse to the extraction time period of $100 \mathrm{~ms}$. The steep negative slope at the very beginning of each curve is to accommodate the long exponential tail of the Boltzmann distribution, which contains few ions in comparison with the number of ions in the vicinity of $E_{i} \sim k_{B} T_{i}^{\circ}$, for instance. The steep negative slopes along with the level at which the slopes become more gentle are governed by the ratio of the ion charge to the ion temperature, $q_{i} /\left(k_{B} T_{i}^{\circ}\right)$. This has an important influence on the extraction of ions of different charge states. As an example, assuming two ion species having the same initial ion temperature, the barrier potential for ejection of a charge of $q_{i}=18$ (e.g., $\mathrm{Ar}^{18+}$ ) has to be reduced to a lower value than that of a charge of $q_{i}=6$ (e.g., $\mathrm{C}^{6+}$ ). This is directly related to the fact that ions of higher charge states experience a deeper trapping potential well. As an additional example, note that in Fig. 5, the optimum function used to release $q_{i}=6$ of a temperature of $50 \mathrm{eV}$ is identical to that of $q_{i}=18$ of $150 \mathrm{eV}$, because they both have the same $q_{i} /\left(k_{B} T_{i}^{\circ}\right)$ ratio.

\section{B. Maxwell-Boltzmann distributions}

According to the equipartition theorem, and for high ion temperature ${ }^{1}$, the kinetic energy of the trapped ions is evenly distributed among all three degrees of freedom. For a short extraction period compared with the ion-ion collision time, it can be assumed that during ejection the ions do not exchange energy. In this special case, since the trap is only open in the axial direction, affecting only one degree of freedom, the ion energy distribution (in the axial direction) may well be represented by a normalized Maxwell-Boltzmann distribution 


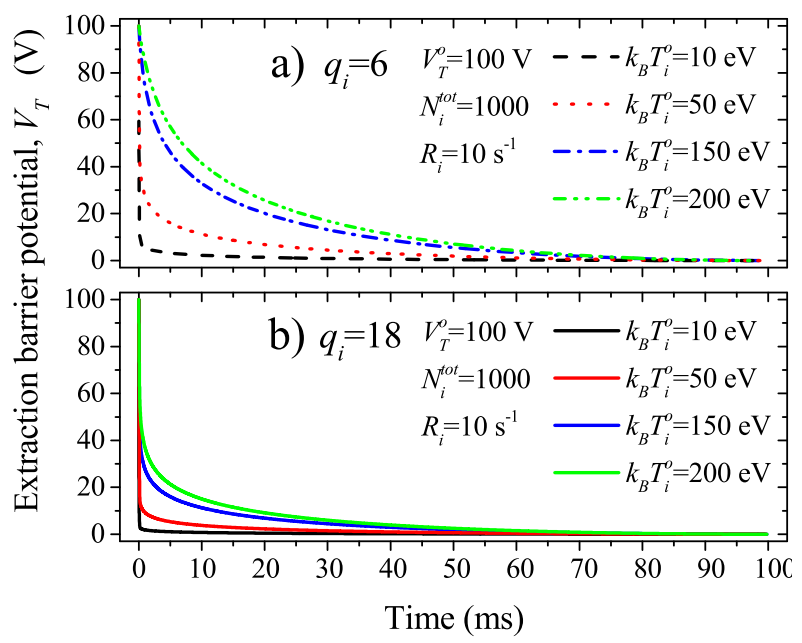

FIG. 6. Optimum functions to eject trapped ions having a Maxwell-Boltzmann energy distribution for one degree of freedom assuming different ion species a) $q_{i}=6$ and b) $q_{i}=18$, and initial ion temperatures.

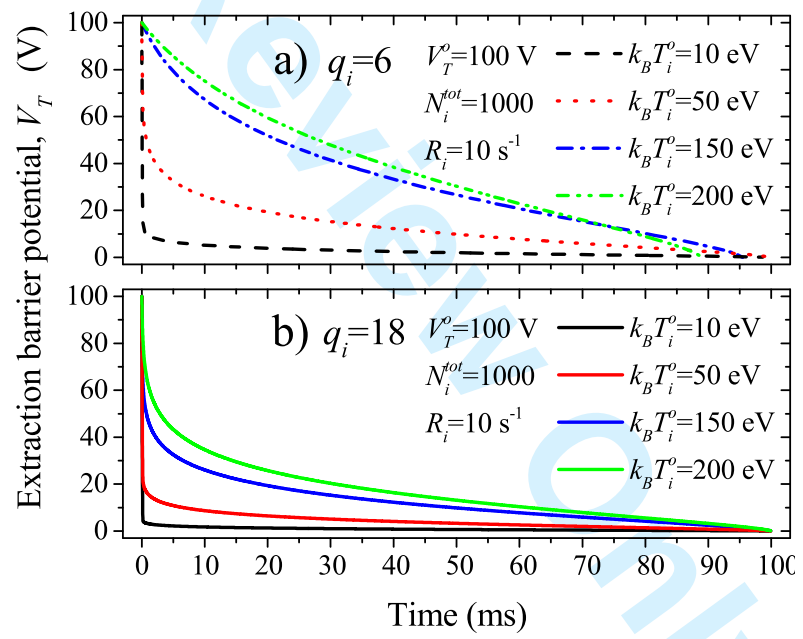

FIG. 7. Optimum functions to eject trapped ions having a Maxwell-Boltzmann energy distribution for three degrees of freedom assuming different ion species a) $q_{i}=6$ and b) $q_{i}=18$, and initial ion temperatures.

for one degree of freedom (1-DoF):

$$
F\left(E_{i}\right)=N_{i}^{t o t} \sqrt{\frac{1}{\pi E_{i} k_{B} T_{i}^{\circ}}} \exp \left[-\frac{E_{i}}{k_{B} T_{i}^{\circ}}\right] .
$$


Substituting Eq. (18) into Eq. (14), integrating, and replacing $E_{i}^{\max }(t)$ with $q_{i} V_{T}(t)$, a simple expression can be obtained for the ideal ejection function:

$$
\frac{R_{i}}{N_{i}^{t o t}} t=-\operatorname{erf}\left(\sqrt{\frac{q_{i} V_{T}(t)}{k_{B} T_{i}^{\circ}}}\right)+C_{1 M B}^{\circ},
$$

where erf is the error function.

A 1-DoF Maxwell-Boltzmann distribution is a more realistic distribution when ion-ion collisions are neglected. For a long extraction period in comparison with the ion-ion collision time, the trapped ions may have time during ejection to distribute their energies among all available degrees of freedom. Still neglecting the influence of ion-ion collisions on the ejection rate, let us assume, as an academic exercise that may be less realistic, that the energy distribution of the trapped ions follows a normalized Maxwell-Boltzmann distribution for three degrees of freedom $(3-\mathrm{DoF})$ as

$$
F\left(E_{i}\right)=2 N_{i}^{t o t} \sqrt{\frac{E_{i}}{\pi}}\left(\frac{1}{k_{B} T_{i}^{\circ}}\right)^{\frac{3}{2}} \exp \left[-\frac{E_{i}}{k_{B} T_{i}^{\circ}}\right] .
$$

Combining Eqs (20) and (14), integrating, and replacing $E_{i}^{\max }(t)$ with $q_{i} V_{T}(t)$, the optimum ejection function for this distribution is:

$$
\frac{R_{i}}{N_{i}^{\text {tot }}} t=2 \sqrt{\frac{q_{i} V_{T}(t)}{\pi k_{B} T_{i}^{\circ}}} \exp \left[-\frac{q_{i} V_{T}(t)}{k_{B} T_{i}^{\circ}}\right]-\operatorname{erf}\left(\sqrt{\frac{q_{i} V_{T}(t)}{k_{B} T_{i}^{\circ}}}\right)+C_{3 M B}^{\circ} .
$$

Solving $V_{T}(t)$ numerically, Figs 6 and 7 show Eqs (19) and (21) plotted for different ion species and temperatures. Overall, the ideal ejection functions of Maxwell-Boltzmann distributions are nearly identical to those obtained previously for Boltzmann distributions. As shown in Fig. 8, which compares results from all three distributions, this statement is particularly true for the 1-DoF distribution. In all cases, the steep negative slopes at the beginning of the extraction period are to match the long high-energy tails of the distributions driven by the exponential terms, which have as argument the same ratio $q_{i} /\left(k_{B} T_{i}^{\circ}\right)$. Unlike the Boltzmann and 1-DoF Maxwell-Boltzmann distributions, the 3-DoF distribution features, however, a faster drop toward the end of the extraction period caused by the $\sqrt{E_{i}}$ multiplicative factor in Eq. (20). Here, it is interesting to mention that for either a high ion temperature or a low ion charge state, or else for both, that is to say, for a small $q_{i} /\left(k_{B} T_{i}^{\circ}\right)$ ratio, all distributions are nearly identical. The resulting optimum functions obtained from Eqs (17), (19), and (21) do not show steep negative slopes at the beginning of the extraction. Particularly, they are very similar to exponential or linear functions (see $q_{i}=6$ in 


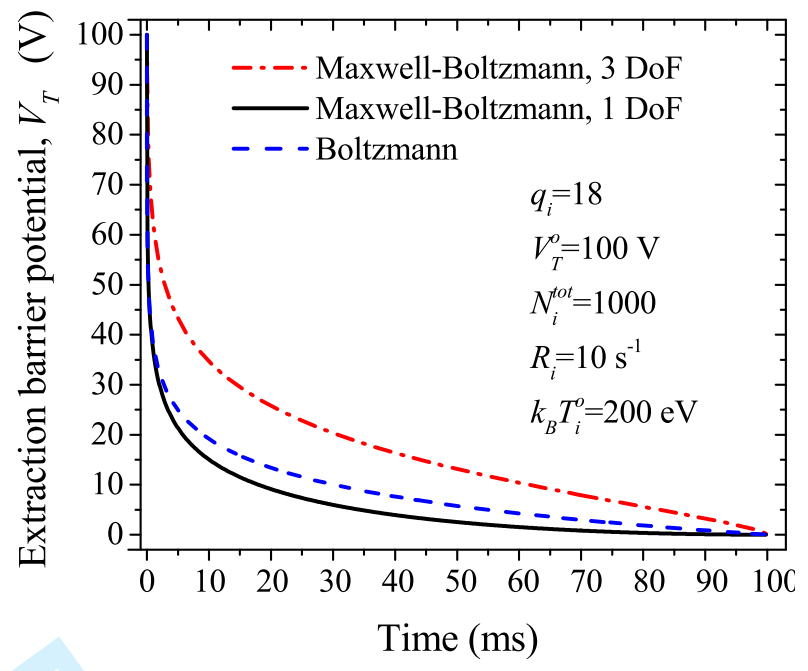

FIG. 8. Optimum ejection functions obtained for a Boltzmann distribution and Maxwell-Boltzmann distributions for one and three degrees of freedom (DoF), assuming the same ion species of $q_{i}=18$ and temperature of $200 \mathrm{eV}$.

Fig. 7), and, as a good approximation, either ones could be used for practical purposes and simplicity to extract ions.

\section{Gaussian distribution}

The spread in energy of singly charged ion beams injected into EBIST is normally small in comparison with the temperature of highly charged ions that have been confined in the trap for long breeding times. However, in very special cases, for very short interaction times of the injected ions with the electron beam (e.g., short breeding times), the energy distribution of the trapped ions may be more adequately represented by a normalized Gaussian distribution such as

$$
F\left(E_{i}\right)=\frac{N_{i}^{t o t}}{\sqrt{2 \pi \sigma_{i}^{2}}} \exp \left[-\frac{\left(E_{i}-E_{a v}\right)^{2}}{2 \sigma_{i}^{2}}\right],
$$

where $\sigma_{i}$ and $E_{a v}$ are the energy spread and average energy of the distribution, respectively. For a Gaussian distribution, the ideal ejection function can be obtained by substituting Eq. (22) into Eq. (14):

$$
\frac{R_{i}}{N_{i}^{t o t}} t=-\frac{1}{2} \operatorname{erf}\left(\frac{q_{i} V_{T}(t)-E_{a v}}{\sqrt{2 \sigma_{i}^{2}}}\right)+C_{G}^{\circ} .
$$

Figure 9 presents ejection functions obtained by numerically solving $V_{T}(t)$ for different ion 


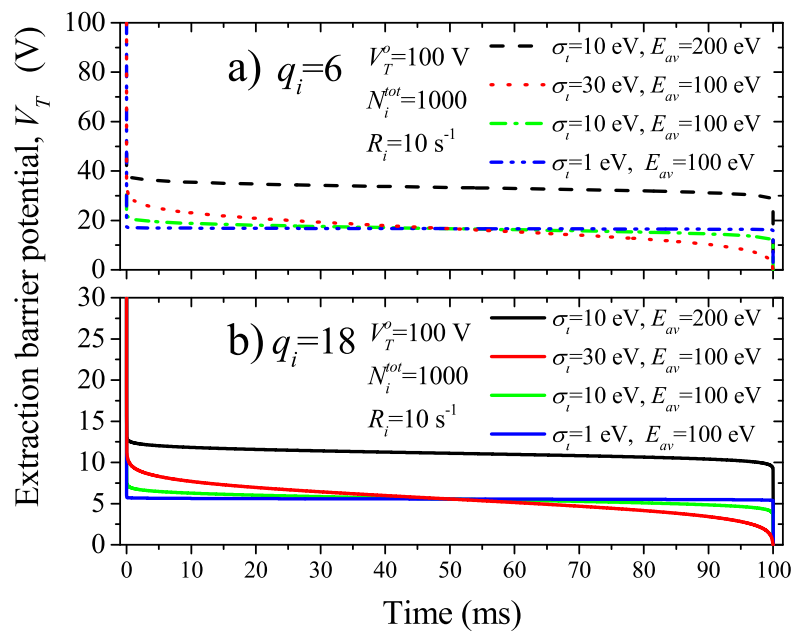

FIG. 9. Optimum functions to eject trapped ions having a Gaussian energy distribution for different ion species a) $q_{i}=6$ and b) $q_{i}=18$, and initial ion temperatures.

species and temperatures. Like for the Boltzmann and Maxwell-Boltzmann distributions, the first segments of the curves shown in Fig. 9 feature steep negative slopes to account for the small number of high-energy ions in the Gaussian distributions. This is followed by a change to gentler negative slopes to accommodate the larger number of ions at the peak of the distributions, with inflection points centered at a value of the barrier potential corresponding to the average energy. Similar to the 3-DoF Maxwell-Boltzmann distribution, the optimum functions of the Gaussian distributions show prominent negative slopes at low energy resulting from the limited number of ions in this energy range. Smaller energy spreads gives rise to gentler slopes for the extraction barrier to be lowered at a reduced rate in order to keep the extraction rate constant.

\section{Experimental distribution}

For any given arbitrary initial energy distribution of the trapped ions, the optimum or ideal function to lower the extraction barrier potential for the release of ions at a constant rate over the extraction period can be deduced using Eq. (14). In the previous sections, the distributions were assumed to be Boltzmann, Maxwell-Boltzmann, and Gaussian distributions. However, the initial ion energy distribution may be unknown. An approach, which may be considered pragmatic, is to decrease this potential with a series of incremental step functions, fine-tuned to generate a square ion time distribution ${ }^{19}$. Another pragmatic ap- 


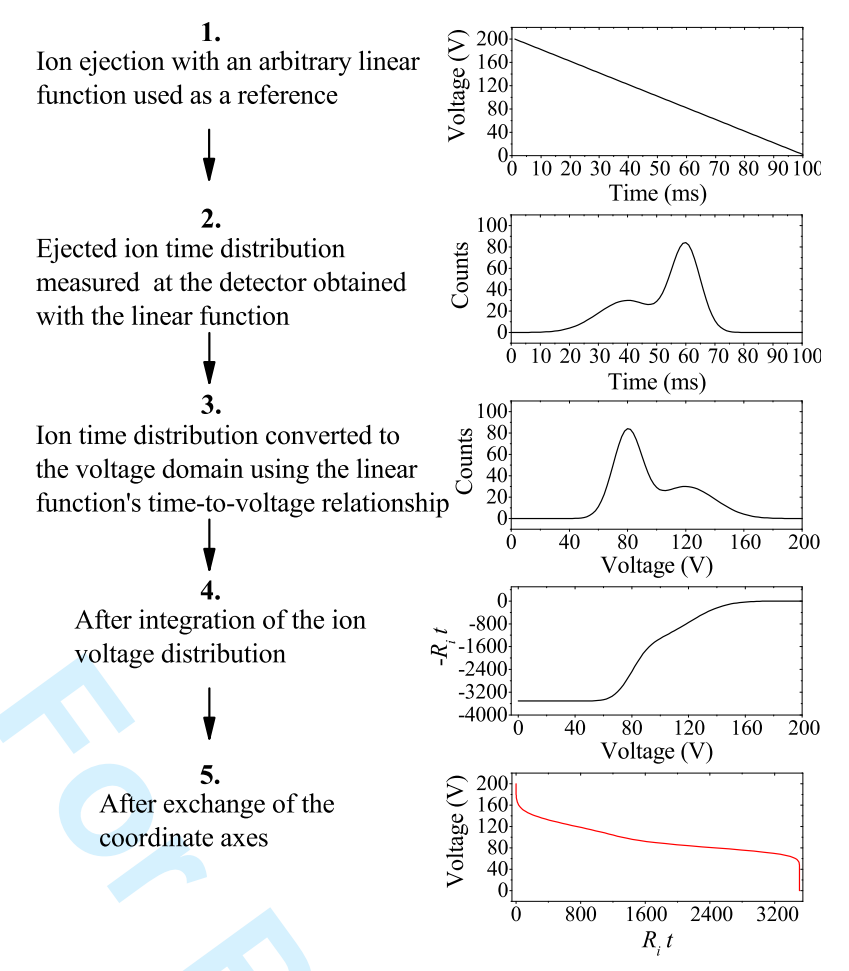

FIG. 10. Procedure to infer the optimum voltage function for ion ejection from an initially unknown ion energy distribution.

proach is to infer the optimum function directly from a time distribution of ejected ions measured at a detector. The procedure to follow is depicted in Fig. 10. The first step in this procedure consists of determining the ion energy distribution. This can be accomplished by ejecting ions to a detector using a known arbitrary function as a reference (e.g., a linear function) and measuring the resulting ejected ion time distribution. In Fig. 10, the ion time distribution is assumed to be a superposition of two Gaussian distributions, as an example. The extraction function being known, the measured time distribution that is in the time domain can then be converted to the voltage domain. For a linear function such as $V_{T}(t)=-\alpha t+V_{T}^{\circ}$, the time domain can be converted with the relation $t=\left(V_{T}^{\circ}-V_{T}(t)\right) / \alpha$. The trapping potential being related to the energy of the ions in the trap through $E_{i}=q_{i} V_{T}$, the time-to-voltage (or energy) conversion yields the energy distribution of the trapped ions. From this step, the procedure simply follows Eq. (14). Numerically integrating the measured experimental ion energy distribution in the voltage domain gives a function which, when plotted, displays $-R_{i} t$ as a function of $q_{i} V_{T}(t)$. Exchanging the axes of the Cartesian coordinates and plotting $q_{i} V_{T}(t)$ as a function of $R_{i} t$, instead, reveal the optimum ejection 
function.

\section{CONCLUSION}

The highly charged ion pulses ejected from EBIST charge breeders at radioactive ion beam facilities can often be too densely distributed for many nuclear-physics detection systems to be able to detect, within each pulse, all ions or events generated by such ions. This publication derives time-dependent functions to open the EBIST's long square axial trapping potential to uniformly spread the release of the ions over the entire extraction period. Although these functions were derived neglecting the effect of ion-ion collisions on the extraction rate, which may not be a good approximation for high ion densities, Eq. (17) has recently produced very satisfactory results at the ReA facility ${ }^{7}$. It has so far allowed the width of ejected pulses to be stretched from $25 \mu$ s (extraction with a step function) up to approximately $80 \mathrm{~ms}$. Systematic studies are currently being conducted. Time-dependent calculations simulating ion extraction with a dynamic opening of the ion trap and incorporating ion-ion collisions have been undertaken. The concepts presented in this work will be included in these simulations.

\section{ACKNOWLEDGMENTS}

The author wishes to thank S. C. S., D. L., S. J. W., as well as the Referees for their comments and suggestions.

1 F. J. Currell and G. Fussmann, IEEE Transaction on Plasma Science, 336 (2005)

2 G. Zschornack, M. Schmidt, and A. Thorn, in CAS-CERN Accelerator School, Ion Sources, edited by R. Bailey (CERN, 2013), p. 165, CERN-2013-007

3 For a recent overview of research conducted with EBIST: Proceedings of the XII International Symposium on Electron Beam Ion Sources and Traps, East Lansing, MI, 18-21 May 2014, edited by A. Lapierre, S. Schwarz, and T. M. Baumann, AIP Conference Proceedings Vol. 1640 (AIP, 2015). 
4 F. Wenander, in CAS-CERN Accelerator School, Ion Sources, edited by R. Bailey (CERN, 2013), p. 351, CERN-2013-007

5 S. Schwarz and A. Lapierre, Rev. Sci. Instrum., 87 02A910 (2016)

${ }^{6}$ F. Wenander, J. Instrum., 5 C10004 (2010)

7 A. Lapierre, S. Schwarz, T. M. Baumann, K. Cooper, K. Kittimanapun, A. J. Rodriguez, C. Sumithrarachchi, S. J. Williams, W. Wittmer, D. Leitner, and G. Bollen, Rev. Sci. Instrum., 85 02B701 (2014)

8 S. Kondrashev, A. Barcikowski, C. Dickerson, P. Ostroumov, S. Sharamentov, R. Vondrasek, and A. Pikin, AIP Conf. Proc., 164054 (2015)

9 A. Lapierre, M. Brodeur, T. Brunner, S. Ettenauer, A. T. Gallant, V. Simon, M. Good, M. W. Froese, J. Crespo López-Urrutia, P. Delheij, S. Epp, R. Ringle, S. Schwarz, J. Ullrich, J. Dilling, Nucl. Instr. Meth. Phys. Res. A, 624, 54 (2010)

10 B. M. Penetrante, J. N. Bardsley, D. DeWitt, M. Clark, and D. Schneider, Phys. Rev. A 43 $4861(1991)$

11 I. V. Kalaginy, D. Küchler, V. P. Ovsyannikovy, and G. Zschornackz, Plasma Sources Sci. Technol. 7441 (1998)

12 R.E. Marrs, Nucl. Instr. Meth. Phys. Res. B 149182 (1999)

13 Y. F. Liu, K. Yao, R. Hutton, and Y Zou, J. Phys. B: At. Mol. Opt. Phys. 383207 (2005)

14 Xiaojun Lu, and F. J. Currell, Phys. Rev. Accel. Beams 12014401 (2009)

15 L. S. Brown and G. Gabrielse, Rev. Mod. Phys. 58233 (1986)

16 K. Blaum, Phys. Rep. 4251 (2006)

17 G. Gabrielse, X. Fei, L. A. Orozco, R. L. Tjoelker, J. Haas, H. Kalinowsky, T. A. Trainor, and W. Kells, Phys. Rev. Lett. 6313601989

18 S. Fogwell Hoogerheide, A. S. Naing, J. M. Dreiling, S. M. Brewer, N. D. Guise, and J. N. Tan, Atoms 3367 (2015)

19 F. Ullman, A. Schwan, G. Zschornack, O. Heid, H. v. Jagwitz-Biegnitz, U. Hagen, Proceedings of IPAC2011, San Sebastián, Spain, 2011. 
$\phi$ : Polar angle

$\theta$ : Azimuthal angle

$\mathbf{v}_{\mathbf{i}}$ : Ion velocity

$v_{i z}: z$-component of the ion velocity vector

$N_{i}^{e j}$ : Number of ejected ions

$N_{i}^{t r}$ : Number of trapped ions

$N_{i}^{\text {tot }}$ : Total number of ions

$i$ : Ion species

$l$ : Ion position

$f\left(v_{i}\right)$ : Ion speed distribution

$L$ : Length of the trapping region

$t$ : Time

B: Magnetic field vector

$E_{i}$ : Ion kinetic energy

$m_{i}$ : Ion mass

$V_{T}(t)$ : Extraction barrier potential/voltage

$\alpha$ : Rate at which the extraction barrier potential is lowered

$q_{i}$ : Ion charge of an ion species $i$

$R_{i}(t)$ : Ion extraction rate

$C^{\circ}$ : Constant depending on initial conditions at $t=0$

$F\left(E_{i}\right)$ : Ion energy distribution

$k_{B}$ : Boltzmann constant

$T_{i}^{\circ}$ : Initial temperature of the trapped ion ensemble

$E_{a v}$ : Average energy of an assumed Gaussian ion energy distribution

$\sigma_{i}$ : Energy spread of an assumed Gaussian ion energy distribution 
FIG. 1. Simplified model and cartoon of an electron beam ion source, which consists of 3 basic components: 1) an electron gun providing a high-current electron beam, 2) an ion trap made with coaxial electrodes sitting in the bore of a solenoid or coils (usually), used to generate a magnetic field of high flux density, and 3) a collector to stop the electron beam. The electron beam is magnetically compressed in the trap's center to a high current density, needed for production of highly charged ions by electron-impact ionization.

FIG. 2. a) Pulsed ion beam injection and extraction scheme: 1. singly charged ions are injected, 2. captured in the axial trapping potential, 3. charge bred to highly charged ions with a Maxwell-Boltzmann energy distribution (the arrows show the ions' velocity vectors), and 4 . released at a constant rate, $R_{i}$, by slowly lowering the collector-side trapping barrier potential with a continuous (time-dependent) function. b) Velocity vector components of two trapped ions in a trap of length $L$.

FIG. 3. Time distributions (calculated with Eq. (6)) of argon ion pulses ejected by lowering the extraction barrier potential with a step time function for a trap length of a) $L=0.01$ $\mathrm{m}$ and b) $L=0.5 \mathrm{~m}$, and ion kinetic energies of 5,50 , and $500 \mathrm{eV}$. Note the difference in time scale between a) and b). For comparison, c) shows the result of a charged-particle trajectory simulation assuming a Maxwell-Boltzmann speed distribution (see text).

FIG. 4. Time distributions of ions ejected with a linear function for different initial ion energy distributions.

FIG. 5. Optimum functions to eject trapped ions having a Boltzmann energy distribution for different ion species a) $q_{i}=6$ and b) $q_{i}=18$, and initial ion temperatures.

FIG. 6. Optimum functions to eject trapped ions having a Maxwell-Boltzmann energy distribution for one degree of freedom assuming different ion species a) $q_{i}=6$ and b) $q_{i}=18$, and initial ion temperatures.

FIG. 7. Optimum functions to eject trapped ions having a Maxwell-Boltzmann energy distribution for three degrees of freedom assuming different ion species a) $q_{i}=6$ and b) 
$q_{i}=18$, and initial ion temperatures.

FIG. 8. Optimum ejection functions obtained for a Boltzmann distribution and MaxwellBoltzmann distributions for one and three degrees of freedom (DoF), assuming the same ion species of $q_{i}=18$ and temperature of $200 \mathrm{eV}$.

FIG. 9. Optimum functions to eject trapped ions having a Gaussian energy distribution for different ion species a) $q_{i}=6$ and b) $q_{i}=18$, and initial ion temperatures.

FIG. 10. Procedure to infer the optimum voltage function for ion ejection from an initially unknown ion energy distribution. 